\title{
Implementing Storage as a Service in Cloud using Network Attached Storage
}

\author{
Sukhdeep Singh \\ M. Tech Student \\ Lovely Professional University \\ Phagwara, India
}

\author{
Sami Anand \\ Assistant Professor \\ Lovely Professional University \\ Phagwara, India
}

\begin{abstract}
Cloud computing is rapidly growing. Still there is need to enhance the cloud computing in terms of availability and security aspects of Cloud service. When we talks about cloud computing, it means we have ability to share the resources through internet. We can store our data on internet space that is provided by service provider. This totally depends upon the service provider that how they store the data and what kind of security they are applying on it. This paper focuses on the cloud storage system. Currently Service Providers are using SAN (Storage area network) as a cloud storage system that is very expensive and need highly trained staff to manage .NAS (Network Attached Storage) has been proposed in this paper to implement "storage as a service" in cloud. NAS is easy to implement and very effective for sharing of data and also in terms of cost. Storage as a service is implemented using NAS and tested in small environment. It shows that it is an effective storage system for cloud in terms of cost and the management of the system.
\end{abstract}

\section{Keywords}

Cloud Computing, NAS, Cloud, API, DAS, Sync.

\section{INTRODUCTION}

Cloud computing is a type of computing in which computer resources are shared and provide the access to end users through internet. Cloud computing provides the resources online to use directly rather than to having local devices and server. Cloud computing also brings the thing in mind that how we can utilize the computational power as much as maximum, Because in cloud computing resources are shared between multiple users and they are using the resources simultaneously so by dong this we are utilizing the computational power. Everything that end-user gets from Service provider that is called "SERVICE". The main advantage of cloud computing is "Pay as per you go" means what kind of service end-user is using is based on the usage. Suppose user is using cloud storage space of 8GB so user will only pay for $8 \mathrm{~GB}$. If he/she wants to expand the space user need to pay as the size of space.

Cloud Computing is a technology that uses the internet and multiple remote servers to maintain data and applications. In cloud computing end-users can uses applications without installation on their own systems and can access their data from anywhere and anytime using internet. Gmail, or Hotmail are Example of cloud computing.

\subsection{Service Model}

Cloud computing is a very vast domain and provides various services to end user. Everything that we get from cloud service provider over internet is called "SERVICE". Services are categorized a below:-
A. SaaS: - SaaS is software as service. As the name suggest service provider provides the virtual copy of particular software for the customer over internet. SP make a web interface that looks like as the virtual copy of that software. End-user can interact with that interface and can do whatever he/she wants without installing it on the local machine. Example of SaaS is "Google docs", Microsoft 360, Photofunia etc.

B. PaaS: - Next service is Platform as a service. In this service end user get the freedom to install their own developed applications on cloud platform provided by Service Provider. Service Provider provides a platform on which end-user can host the application or software. Example of PaaS is Googleapp engine, Microsoft windows azure platform.

C. IaaS: - IaaS is an infrastructure as a service in this Service Providers gives the hardware, networking resources to end user. Mainly storage comes under this section. In storage end-user get the benefits of storage to store the data on cloud. In this data is managed by service provider, end-user does not aware where the data is actually stored. Examples of IaaS are: - Google Drive, Sky drive etc.

\section{STORAGE SYSTEM IN CLOUD}

Cloud storage systems are consist of multiple storage device that are available all the time .Once the users store their data in cloud it makes multiple copies of data on multiple storage devices that can be located on different-different geographical area this is called "Data Redundancy" that is the main motive of cloud computing. Cloud computing ensures that once data is stored in cloud it must be available to end user anytime and from anywhere whether there is any failure of Server it should still be served by another server. If we talks about traditional Storage systems Like DAS (Direct Attached Storage) Systems [5].

\subsection{DAS}

In DAS typically Service Providers uses Storage servers that are responsible to save and retrieve the file from the harddisks that are directly attached to the server. So in this scenario service provider need to establish separate server or if SP does not use separate servers then it increase the overhead of the server because server is responsible to serve the client request and also for saving the data in storage. Now in the DAS scenario if service provider wants to increase the storage space then more hard disks need to be attached that not only creates the overhead of the server but also decrease the operation time to fetch the data from the hard-disks .On storage system that is using DAS, RAID is required to be implemented to create redundancy in case of hard-disk failure. 
The Main disadvantage of the DAS storage system is that it is not possible to share storage with another server so it means if the server crashes then the storage system is no more available to use [2].

\subsection{Storage Area Network}

Storage area network storage is very famous in cloud computing. Most of the Service providers are using SAN systems to provide the services. SAN is a pool of storage where multiple storage devices can be connected to each other via local network or fiber cables. SAN stores data in the form of blocks. Multiple storage devices are put together and the seams as a single storage system.

\subsection{NAS}

Network Attached Storage systems are the dedicated storage systems that are connected within the network .NAS storage works with an IP address so it can be easily shared with the multiple server in cloud. NAS can be used as primary storage in cloud computing and also can be used to make redundancy of the data. Because NAS works separately that's why it decreases the overhead of the server. NAS provides the file systems like NFS, CIFS that can be easily shared across the network [2]. Now suppose we have multiple NAS storage in our cloud and multiples servers are there. Now the responsibility of server is only to serve the request of end-user they are not taking the headache of data storage because all the data is stored and managed by the NAS systems. Server will only take the data from users and pass it to the NAS system and whenever the data is required server will send a request to the NAS system and it will return the data. NAS works on network as separate machine it is capable to provide FTP, HTTP service so we don't need to establish separate FTP server as usually Service providers do [2].

\section{METHODOLOGY}

In this paper NAS system is purposed to implement storage as a service. In this system organization can use NAS system as primary storage as well as backup systems to make cloud fault tolerant in case of any server or storage system failure. Here is the Architecture of the developed cloud system to provide the storage service to end users by implementing the web interface.

A service can be implemented in two ways:-

API- API is an application programming interface .It is used to serve the services to end user by installing the desktop application on user's system. End-user don't need to browse the web they can simply use this software and it will automatically connect to that web service and user can access their files.

WEB INTERFACE: - Web interface provides full control on data. User need to browse the particular website to access the data from anywhere and anytime. In API system user need to install the software on system then he/she will be able to use the services.

\subsection{System Architecture}

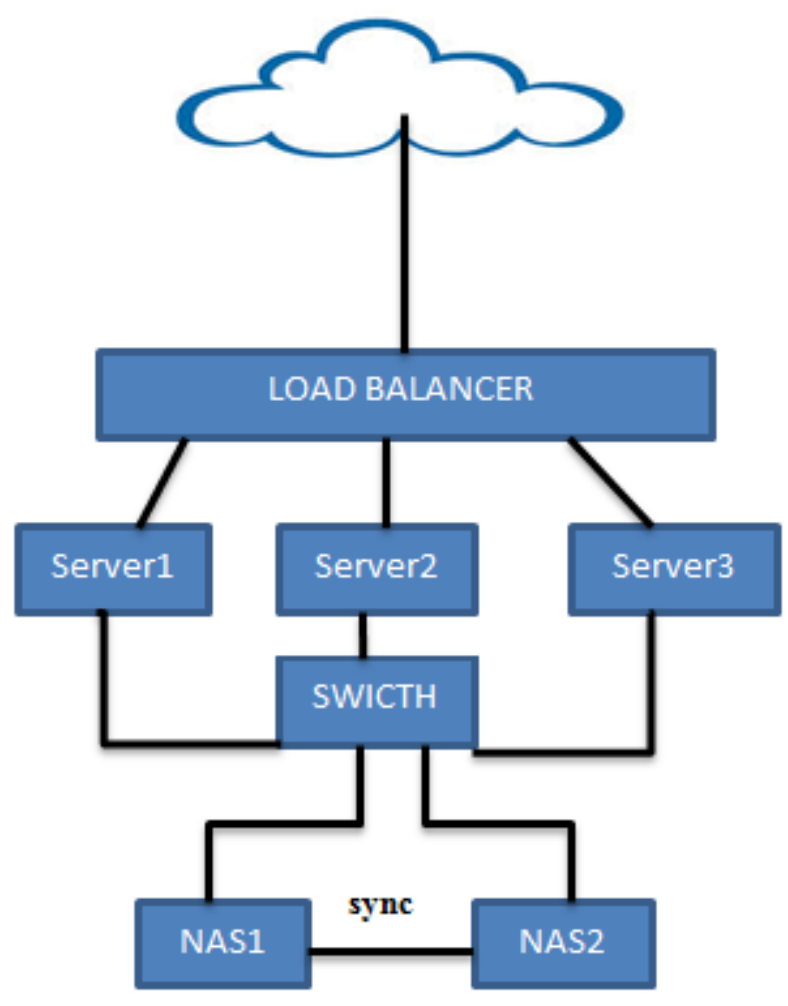

\subsection{Load Balancer}

There are three server are running simultaneously that are serving the request of en-user. So in this load must be balanced between servers so that overhead of the server can be decreased. Multiple servers also help to make service available all the time. Suppose server1 failed or crashed and it is no more available so in this case server 2 and server 3 are available to take place of server1. The role of Load balancer is to divide the load into three servers and it also checks that all servers are online and if it founds that any of the server is offline it automatically stops sending request to that server. In this scenario suppose server1 crashes then the incoming request will not go to the server1. Load balancer will transfer these requests to server 2 and server 3 until server 1 is available.

In this System server are responsible only to server the request or we can say servers are acting as management server. They will get the data from users and send it to the NAS system. NAS provides FTP service and using this service, servers can interact with NAS.

\section{IMPLEMENTATION}

NAS systems are used in this cloud system. There are two NAS are used that are synchronized to each other means data redundancy is there. It means if any of the NAS crashed still users are able to get back their data. This system is developed on small scale. This can be implemented on large scale by adding more NAS systems and more servers.

Storage As a Service -- Here this cloud system provides Storage as a service to end users. To provide services to end user a web interface has been developed using .net c\# and Microsoft Access Database is used as backend database to store user's information. So the web interface is running on each server so to maintain the session of the user a 'State Server Session" is used. A separate server is storing the session state of the user. This is very important in cloud 
because if user is logged on one server and after that load balancer transfer that user to another server it means another server need to recreate the session for that user that will be totally wastage of memory. That "why in this system "State server session" is maintained by separate server. In this case if load balancer transfer the request or shift the user to another server then the second server will fetch the session data from state server and will able to provide the service to end user.

\section{TESTING OF THE SYSTEM}

This system is tested in small environment and data is collected from different NAS system and determined that how much load is running on the NAS systems when the end users were uploading their files in the cloud. This testing is done via internet not on LAN. So the testing of this system is real time testing. Results are given below of NAS systems total users were 50. They all are uploading data in cloud simultaneously.

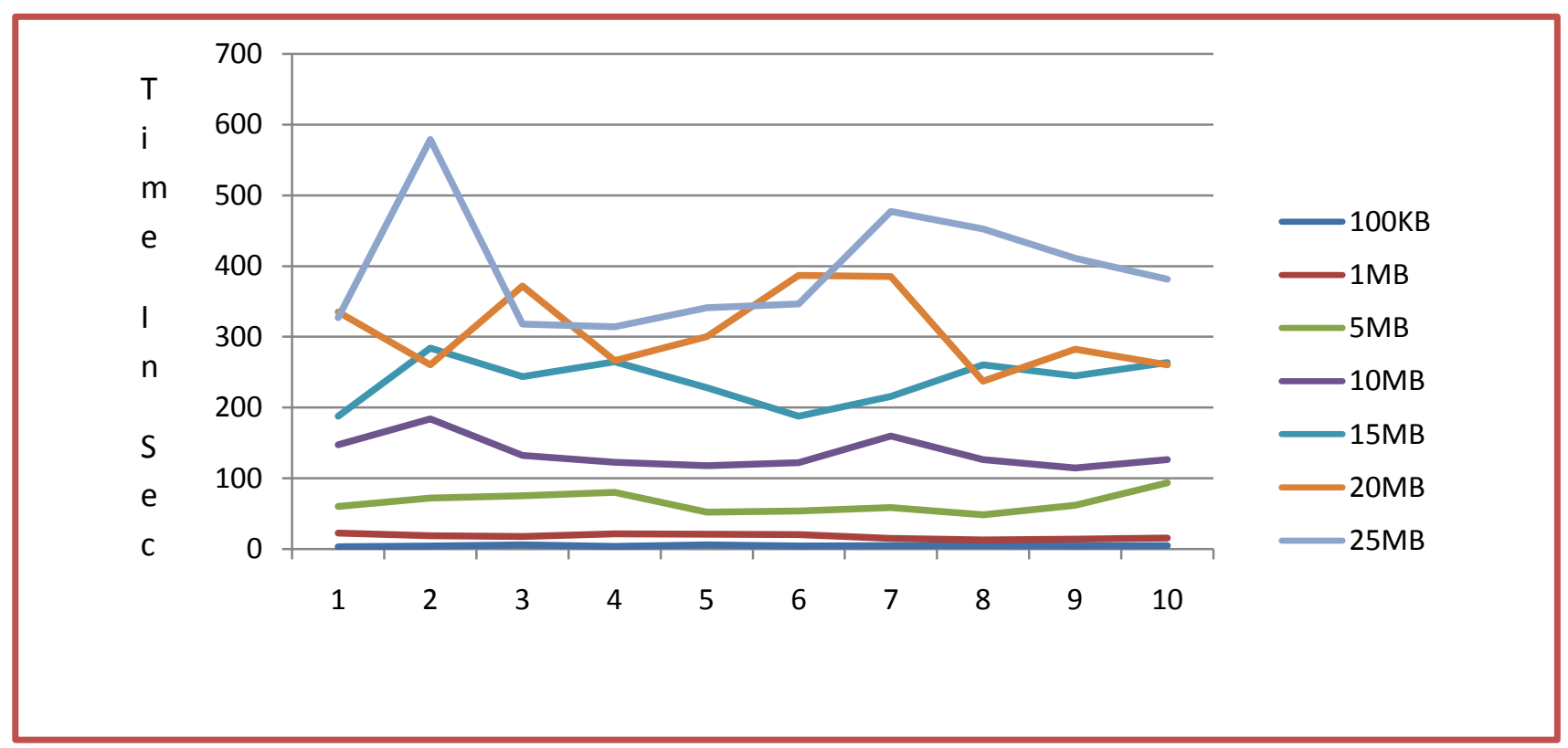

Figure 1: File upload time Graph

This graph shows that how much time this system takes to migrate the file from client side to the NAS system this purposed system is evaluated in small environment and results are gathered by uploading multiple files of the same size. Here in the graph we have files of size $100 \mathrm{~KB}, 1 \mathrm{MB}, 5 \mathrm{MB}, 10 \mathrm{MB}, 15 \mathrm{MB}, 20 \mathrm{MB}, 25 \mathrm{MB}$ respectively and uploaded 10 files of each 10 times. It means 10 files of $100 \mathrm{~KB}$ uploaded 10 times into the system to get the average time. Here in the graph a vertical line shows the upload time in seconds and horizontal line shows the how many times files were uploaded. In this evaluation 10 times same file uploaded to get average time. So in the graph if we see when we are uploading $100 \mathrm{~KB}$ of files 10 times we don't see huge fluctuation, its smooth line it means we are getting almost equal time on every try. And if we see in files of $25 \mathrm{MB}$ then in starting it gives fluctuation but after it's time line get smooth.

AVG.

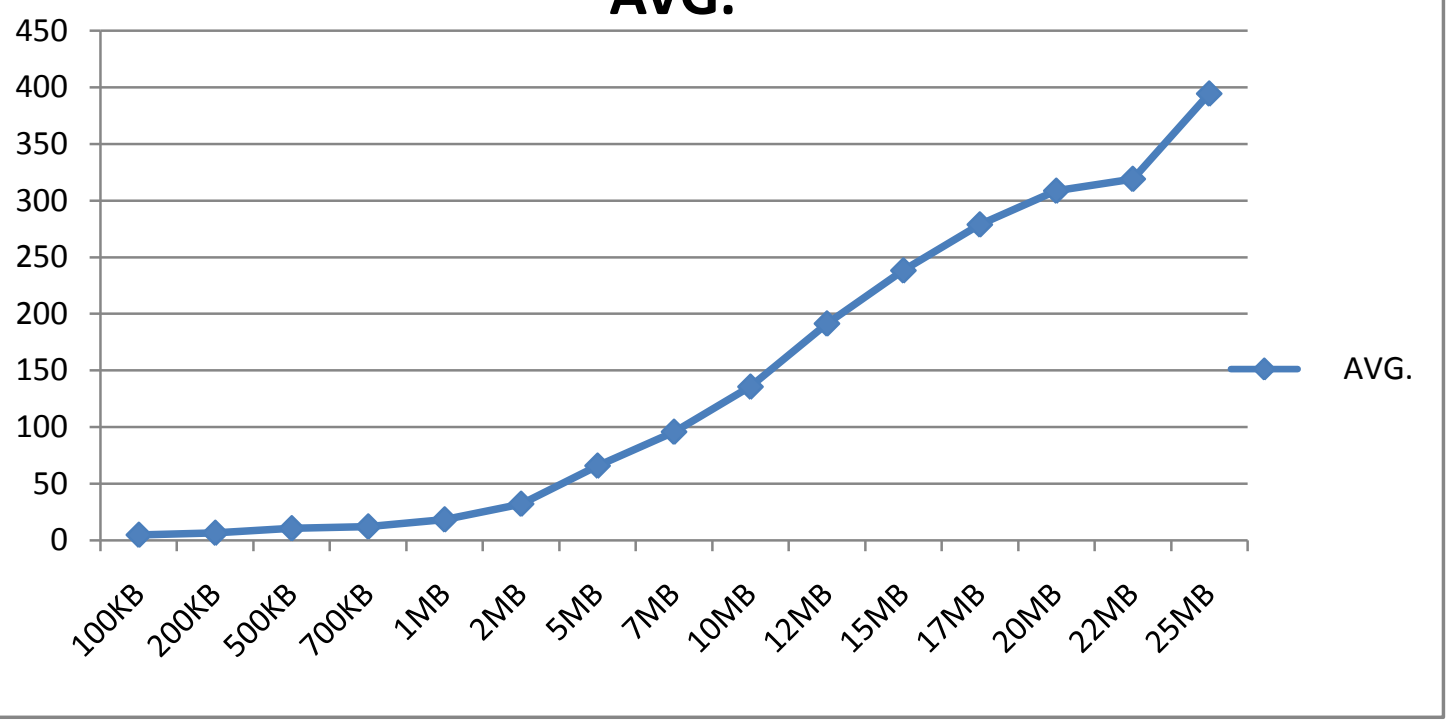

Figure 2: Average Time Graph 
Average time graph shows the average time taken to upload files in the system. Here we can say that the file upload time is not directly proportional to the size of the file. If we look into the graph when we are uploading files of size $100 \mathrm{~KB}$ it takes average time of 4.59 seconds and if we see the $1 \mathrm{MB}$ file it takes 18.18 seconds to upload. Here the part of interest is that if we pay attention to file size here the files size is increased 10 times, means first we are uploading $100 \mathrm{~KB}$ and now
Uploading $1 \mathrm{MB}$. It's actually more than 10 times and if we see the average file upload time then for $100 \mathrm{~KB}$ it is $4.59 \mathrm{~s}$ and for $1 \mathrm{MB}$ it is $18.18 \mathrm{~s}$. If the file size in increased 10 times so that the time should also increase with the same ratio but here the time is increasing with the multiplication factor of 4 means the expected factor is 10 but we are getting 4. It means time is increasing half of the expectations.

Table 1: Upload time

\begin{tabular}{|l|r|r|r|r|r|r|r|r|r|r|r|r|}
\hline & 1 & 2 & 3 & 4 & 5 & 6 & 7 & 8 & 9 & 10 & AVG. \\
\hline $100 \mathrm{~KB}$ & 3.169 & 4.326 & 5.774 & 3.762 & 5.998 & 4.214 & 4.85 & 4.664 & 4.353 & 4.806 & 4.5916 \\
\hline $200 \mathrm{~KB}$ & 7.924 & 3.923 & 6.121 & 7.558 & 6.482 & 5.921 & 10.472 & 4.781 & 5.699 & 4.545 & 6.3426 \\
\hline $500 \mathrm{~KB}$ & 7.781 & 11.058 & 10.379 & 8.67 & 14.096 & 11.577 & 10.378 & 9.289 & 11.096 & 9.559 & 10.3883 \\
\hline $700 \mathrm{~KB}$ & 12.034 & 11.247 & 8.515 & 10 & 11.698 & 14.754 & 11.052 & 11.385 & 10.102 & 18.578 & 11.9365 \\
\hline $1 \mathrm{MB}$ & 22.87 & 18.968 & 17.8 & 21.679 & 21.388 & 20.847 & 15.311 & 13.165 & 14.179 & 15.601 & 18.1808 \\
\hline $2 \mathrm{MB}$ & 40.719 & 40.465 & 29.698 & 41.731 & 35.454 & 36.203 & 25.226 & 23.914 & 23.981 & 22.629 & 32.002 \\
\hline $5 \mathrm{MB}$ & 60.218 & 72.394 & 75.084 & 80.125 & 52.415 & 53.743 & 58.753 & 48.665 & 62.03 & 93.475 & 65.6902 \\
\hline $7 \mathrm{MB}$ & 127.928 & 121.896 & 81.7 & 93.955 & 86.722 & 76.323 & 96.445 & 88.819 & 99.943 & 82.984 & 95.6715 \\
\hline $10 \mathrm{MB}$ & 147.656 & 184.064 & 132.573 & 122.911 & 118.123 & 122.486 & 159.64 & 126.548 & 114.771 & 126.382 & 135.5154 \\
\hline $12 \mathrm{MB}$ & 139.85 & 167.442 & 194.548 & 170.985 & 242.749 & 250.787 & 188.767 & 223.75 & 154.165 & 180.355 & 191.3398 \\
\hline $15 \mathrm{MB}$ & 187.871 & 284.332 & 244.098 & 265.106 & 228.256 & 187.847 & 215.625 & 260.629 & 244.985 & 263.927 & 238.2676 \\
\hline $17 \mathrm{MB}$ & 200.608 & 226.283 & 269.033 & 258.645 & 343.183 & 338.264 & 284.049 & 276.523 & 219.625 & 372.696 & 278.8909 \\
\hline $20 \mathrm{MB}$ & 335.523 & 260.573 & 371.84 & 266.131 & 300.467 & 387.076 & 385.596 & 237.165 & 282.556 & 260.2 & 308.7127 \\
\hline $22 \mathrm{MB}$ & 289.834 & 280.208 & 274.653 & 398.779 & 316.285 & 299.415 & 332.167 & 320.5 & 327.82 & 351.919 & 319.158 \\
\hline $25 \mathrm{MB}$ & 326.722 & 578.93 & 317.705 & 314.055 & 340.761 & 346.263 & 476.978 & 452.578 & 410.844 & 381.154 & 394.599 \\
\hline
\end{tabular}

Let's compare the file upload time with first file that uploaded in the system that is of $100 \mathrm{~KB}$ and it takes 4.59 seconds to upload. Now we will check that if we increase the file size how much time of upload file increases.

Table 2: Upload Average Time

\begin{tabular}{|l|l|l|l|}
\hline $\begin{array}{l}\text { Size of } \\
\text { the File }\end{array}$ & $\begin{array}{l}\text { Average } \\
\text { time(Seconds) }\end{array}$ & Increased size & $\begin{array}{l}\text { Increased } \\
\text { time }\end{array}$ \\
\hline $1 \mathrm{MB}$ & 18.18 & 10 times & 4 times \\
\hline $2 \mathrm{MB}$ & 32.02 & 20 times & 6.97 times \\
\hline $10 \mathrm{MB}$ & 135.51 & $\begin{array}{l}100 \\
\text { times(app.) }\end{array}$ & 29.52 times \\
\hline $20 \mathrm{MB}$ & 308.71 & $\begin{array}{l}200 \quad \text { times } \\
(\text { app. })\end{array}$ & 67.25 times \\
\hline $25 \mathrm{MB}$ & 394.59 & $\begin{array}{l}250 \text { time } \\
\text { (app.) }\end{array}$ & 85.96 times \\
\hline
\end{tabular}

\section{CONCLUSION}

In this system storage as a service is implemented by using three servers and two Network Attached Storage devices. No storage server in used in this system all the data is being stored in NAS systems. Both NAS systems are acting as primary storage as well as backup systems because both are synchronized to each other if one fails then data can be restored from second one. Test results of the system shows that system is feasible and can be implemented same this on the large scale to hold the huge amount of data. Here the one thing is that speed of data transfer is not directly proportional to the file size but it depends on the network speed.

\section{REFRENCES}

[1] Hay Brian, Nance Kara, Bishop Matt (2011) "Storm Clouds Rising: Security Challenges for IaaS Cloud Computing", Proceedings of the 44th Hawaii International Conference on System Sciences 2011.

[2] Hsu Wen-Feng, Luo Guo-Heng, Yuan Shyan-Ming, Tsai Ching-Tsorng (2012) "Constructing Private Cloud Storage Using Network Attached Storage" 9th International Conference on Ubiquitous Intelligence and Computing and 9th International Conference on Autonomic and Trusted Computing.

[3] Yang Jianfeng and Chen Zhibin (2010) "Cloud Computing Research and Security Issues", Computational Intelligence and Software Engineering (CiSE), International Conference 2010, Wohan.

[4] Liu Hao and Dezhi Han (2011) "The study and design on secure-cloud storage system".

[5] Gurudatt Kulkarni and Rani Waghmare (2012) "Cloud Storage Architecture", 7th International Conference on Telecommunication Systems, Services, and Applications (TSSA).

[6] Yubo Tan and Xinlei Wang "Research of Cloud Computing Data Security Technology". 